\title{
Modelling of Theoretical Surface Roughness for Two-Dimensional Vibration-Assisted Machining
}

\author{
Y. Gao \\ The State Key Lab of Digital Manufacturing Equipment and \\ Technology \\ School of Mechanical Science and Engineering \\ Huazhong University of Science and Technology \\ China
}

\author{
R.L. Sun \\ The State Key Lab of Digital Manufacturing Equipment and \\ Technology \\ School of Mechanical Science and Engineering \\ Huazhong University of Science and Technology \\ China
}

\begin{abstract}
Vibration-assisted machining (VAM) has been proposed for tens of years and attracts a lot of attentions due to its unique advantages on improving the machining quality of a range of materials, especially intractable materials like titanium and nickel alloys. VAM combines conventional machining (CM) with high-frequency, small-amplitude tool vibrations. This paper presents a customized tool designed for two-dimensional (2D) VAM and reveals its movement rules. A simplified surface generation model is developed to predict the theoretical surface roughness in two-dimensionalVAM, and the model is verified using finite element analysis software. All the influential parameters on theoretical surface roughness using the customized tool are analysed over a wide range of values. This paper provides the theoretical basis for the selection of suitable cutting parameters and vibration parameters before machining.
\end{abstract}

Keywords- vibration-assisted machining; surface roughness

\section{INTRODUCTION}

In the recent years, a wide range of new alloys and composite materials has been applied in aerospace applications, such as titanium and nickel alloys [1]. Due to their high toughness and work hardening behaviours, machining of these alloys is generally extremely difficult [2]. Conventional machining (CM) of these alloys causes high cutting temperatures, large cutting forces and subsequent fast tool wears. These problems eventually lead to the deterioration of machining quality. Vibration-assisted machining (VAM) is proved effective to solve these problems. It adds highfrequency, small-amplitude tool vibrations to the conventional cutting motion of the tool [3]. In 2D VAM, an elliptical vibration locus of tool is generated during the machining process. For appropriate combinations ofnominal cutting speed and tool vibration speed, the tool periodically contacts and loses contact with workpiece and chips. This intermittent machining process brings a lot of advantages, which include reduced tool forces, extended tool lives, reduced surface roughness, improved form accuracy and suppression of burr formation [3].

Some characteristics of 2D VAM are fundamentally different from CM [4]. The existence of vibration cusps on the machined surface along nominal cutting direction is a typical characteristic. The cusps are generated by the overlapping of elliptical vibration locus, and reflect the surface roughness of machined surface. Surface roughness is a significant parameter for evaluating the surface quality after machining and it has tremendous effects on the performances of components, such as fatigue lives [5]. Therefore, in order to better understand the mechanism of 2D VAM and predict surface roughness, a kinematics analytical model is developed in this study to predict the theoretical surface roughness in 2D VAM.

The remainder of the paper is organised as follows. Section 2 a customized tool for 2D VAM is designed and analysed; section 3 presents the development of the theoretical surface roughness model, and verifies the model using AdvantEdge FEM software; section 4 analyses the parameters that affect the theoretical surface roughness.

\section{DeSign OF A CUSTOMIZED TOOL FOR 2D VAM}

A customized tool is designed for 2D VAM, as shown in fig.1. The inserter is fixed onto the head of the tool by a screw. Two parallel arranged piezoelectric transducers (PZTs) are pasted between the head and the connection. The bolt and nut are used to preload the PZTs. To make the assembly of the bolt easily, the slot in the middle of the head must be designed longer than the bolt. The existence of the slot can effectively decrease the crosstalk between two PZTs. The connection connects the taill, with the rest parts of the tool by four screws, and the tail can be fixed on the tool holder, the same as ordinary tools. The overall tool is designed approximate symmetrical in structure and miniature in size.

When energized with sinusoidal voltages, the PZTs expand and contract periodically. The phase difference between two sinusoidal voltages applied to the PZTs leads the tool tip to vibrate in two directions. For kinematics analysis, the head is simply modelled as a T-shaped bar [6], where $l$ and $h$ are basic dimensional parameters of the tool. According to the previous study of the author, the movement rules of the tool tip are expressed as eqn(1), where $A$ represents the vibration amplitudes of the PZTs and $\varphi$ represents the phase difference between two sinusoidal voltages and it shows the tool tip can move along a standard elliptical locus. 


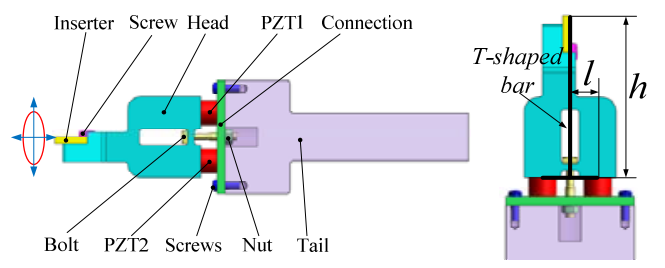

FIGURE I. OVERALL DESIGN OF THE TOOL.

$$
\frac{x^{2}}{\left[A \frac{h}{l} \sin \frac{\varphi}{2}\right]^{2}}+\frac{y^{2}}{\left[A \cos \frac{\varphi}{2}\right]^{2}}=1
$$

\section{MODELLING OF THEORETICAL SURFACE ROUGHNESS}

In 2D VAM, two-dimensional vibration movement of the tool is superimposed with the movement of workpiece. Consider an ideal tool whose tool edge is assumed to be perfectly sharp, its tool tip moves along a consecutive spiral locus, and generates a specific surface waviness, as shown in fig. 2(a). After machining, periodical vibration marks or cusps are left on the machined surface along the nominal cutting direction. The height of the cusps is considered to be the value of theoretical surface roughness Rth. Because the vibration frequency applied in VAM is quite high, the tool moves only a short distance in each vibration cycle. So it is possible to simplify the spiral locus with a set of standard ellipses, as shown in fig. 2(b), the distance between two adjacent ellipses is set equal to the distance that the tool moves in each vibration cycle along the nominal cutting direction. Quite similar surface waviness is generated using the simplified locus compared with the true spiral locus, and it is within the margin of error to deduce the theoretical surface roughness model based on this simplified locus.

(a)

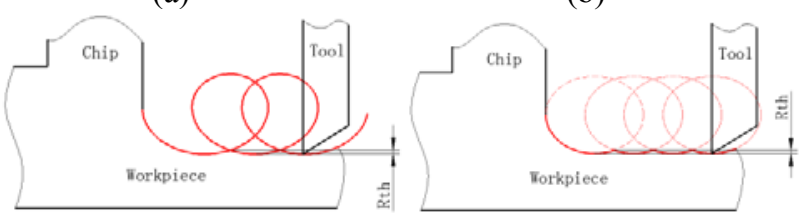

FIGURE II. SURFACE GENERATION MODEL.

However, the tool edge of the tool can never be perfectly sharp, meaning that its radius cannot be equal to zero. Actually, the tool edge radius is usually at micron-level, the same as the vibration amplitudes in VAM. The effect of tool edge radius on the surface generation process cannot be neglect, and the modelling of the theoretical surface roughness should take tool edge radius into consideration. Fig. 3(a) shows the surface generation process in 2D VAM using a round tool edge, whose radius is symbolize as $r$. The dashed line represents the movement locus of the tool edge bottom point. It is a part of standard elliptical locus. The dotted line represents the movement locus of the tool edge centre point. It can be achieved by translating the dashed line upwards by a distance of $r$. The continuous line represents the final machined surface. It is the envelope line of a set of dashed circles which represent the positions of the round tool edge in machining. The theoretical surface roughness is also showed in fig. 3(a).

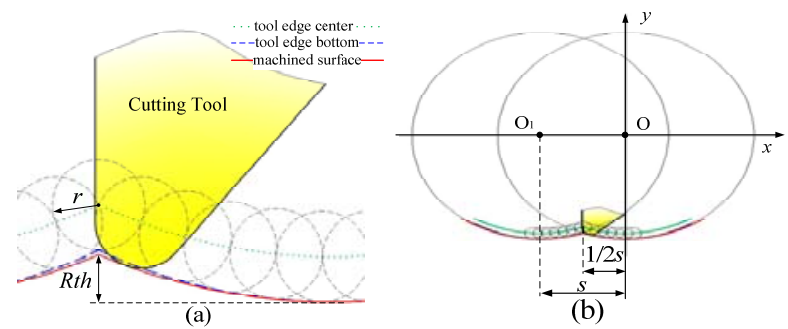

FIGURE III. SURFACE GENERATION MODEL CONSIDERING TOOL EDGE RADIUS

To calculate the theoretical surface roughness, a rectangular coordinate system is established, as shown in fig. 3 (b).Ellipse $\mathrm{O}$ and ellipse $\mathrm{O}_{1}$ represent the movement locus of the tool edge bottom point in two adjacent vibration cycles. The distance between $\mathrm{O}$ and $\mathrm{O}_{1}$ is symbolized as $s$, derived by eqn(2).

$$
s=v T=\frac{v}{f}
$$

The locus of the tool edge centre point is achieved by translating ellipse $\mathrm{O}$ along y direction, and the corresponding locus equation is expressed as eqn(3). Then the curve family of the dashed circles can be expressed as eqn(4).

$$
\begin{aligned}
& \left\{\begin{array}{l}
x=a \cos t \\
y=r+b \sin t
\end{array} \quad t \in\left[\pi, \frac{3}{2} \pi\right]\right. \\
& (x-a \cos t)^{2}+(y-r-b \sin t)^{2}=r^{2}
\end{aligned}
$$

Construct a function:

$$
F(x, y, t)=(x-a \cos t)^{2}+(y-r-b \sin t)^{2}-r^{2}(3)
$$

The locus equation of the envelope line can be derived by solving eqn(6), and it is finally expressed in the form of parametric equation as eqn(7).

$$
\begin{gathered}
\left\{\begin{array}{l}
F(x, y, t)=0 \\
\frac{\partial F(x, y, t)}{\partial t}=0
\end{array}\right. \\
\left\{\begin{array}{l}
x=a \cos t-\frac{r b}{\sqrt{b^{2}+a^{2} \tan ^{2} t}} \\
y=r+b \sin t-\frac{\text { ratant }}{\sqrt{b^{2}+a^{2} \tan ^{2} t}}
\end{array} \quad t \in\left[\pi, \frac{3}{2} \pi\right](5)\right.
\end{gathered}
$$

Due to the symmetry of the envelope line, the x-coordinate of the cusp is $-1 / 2 \mathrm{~s}$. The corresponding parameter $t^{*}$ for the cusp is achieved by solving eqn(8), and then the theoretical surface roughness can be expressed as eqn(9).

$$
\begin{gathered}
a \cos t-\frac{r b}{\sqrt{b^{2}+a^{2} \tan ^{2} t}}=-\frac{1}{2} s \\
R_{t h}=r+b\left(1+\sin t^{*}\right)-\frac{r a \tan t^{*}}{\sqrt{b^{2}+a^{2} \tan ^{2} t^{*}}}
\end{gathered}
$$


The correctness of the theoretical surface roughness model is verified using AdvantEdgeFEM software. When semi-major axis length $a$ changes from $20 \mu \mathrm{m}$ to $50 \mu \mathrm{m}$, the calculated surface roughness value changes from $3.9985 \mu \mathrm{m}$ to $0.8335 \mu \mathrm{m}$, under the circumstances that $b$ equals to $10 \mu \mathrm{m}, r$ equals to $20 \mu \mathrm{m}, v$ equals to $50 \mathrm{~m} / \mathrm{min}$ and $f$ equals to $20 \mathrm{kHz}$. Fig. 4 shows the machined surface becomes smoother with the increase semi-major axis.
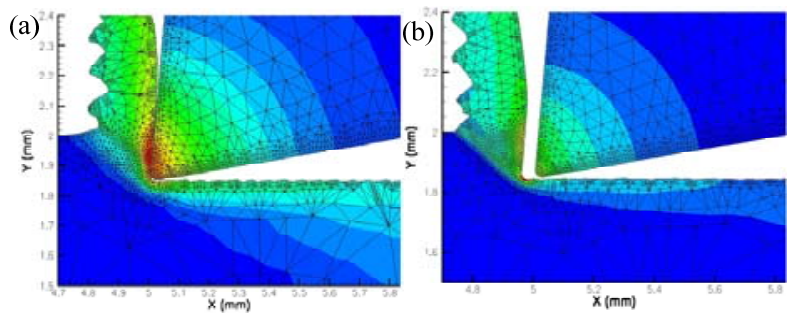

FIGURE IV. SURFACE PROFILES FOR (A) A=20MM AND (B) A=50MM.

When vibration frequency $f$ changes from $15 \mathrm{kHz}$ to $25 \mathrm{kHz}$, the calculated surface roughness value changes from $4.0359 \mu \mathrm{m}$ to $1.3216 \mu \mathrm{m}$, under the circumstances that $a$ equals to $30 \mu \mathrm{m}, b$ equals to $10 \mu \mathrm{m}, v$ equals to $50 \mathrm{~m} / \mathrm{min}$ and $r$ equals to $20 \mu \mathrm{m}$. Fig. 5 shows the machined surface becomes smoother with the increase vibration frequency.
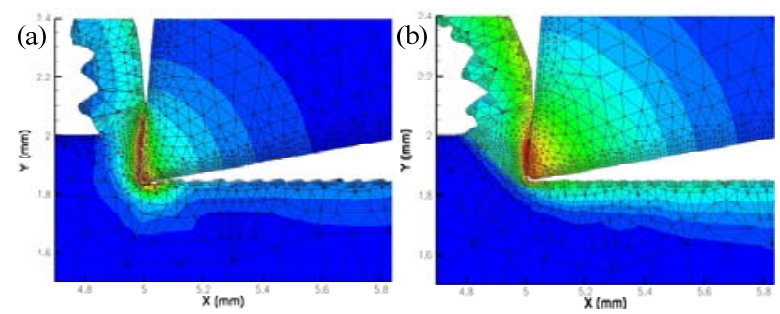

FIGURE V. SURFACE PROFILES FOR (A) F=15KHZ AND (B) F=25KHZ.

\section{ANALYSIS OF SURFACE ROUGHNESS USING THE CUSTOMIZED TOOL}

Fig. 6 shows how various parameters affect the theoretical surface roughness, and their value ranges are listed in Table 1.

TABLE I. VALUE RANGES OF THE INFLUENTIAL PARAMETERS.

\begin{tabular}{|c|c|c|c|c|c|c|c|}
\hline NO. & $\boldsymbol{A}(\boldsymbol{\mu \mathbf { m } )}$ & $\begin{array}{c}\boldsymbol{h}(\mathbf{m m} \\
\mathbf{)}\end{array}$ & $\boldsymbol{l}(\mathbf{m m})$ & $\boldsymbol{\varphi}(\mathbf{r a d})$ & $\boldsymbol{r}(\boldsymbol{\mu \mathbf { m } )}$ & $\boldsymbol{f}(\mathbf{k H z})$ & $\boldsymbol{v}(\mathbf{m} / \mathbf{s})$ \\
\hline (a) & $5 \sim 15$ & 80 & 40 & $\pi / 2$ & 7 & 20 & 0.3 \\
\hline (b) & 10 & $\begin{array}{c}60 \sim 10 \\
0\end{array}$ & 40 & $\pi / 2$ & 7 & 20 & 0.3 \\
\hline (c) & 10 & 80 & $20 \sim 60$ & $\pi / 2$ & 7 & 20 & 0.3 \\
\hline (d) & 10 & 80 & 40 & $\pi / 2 \sim 5 \pi / 6$ & 7 & 20 & 0.3 \\
\hline (e) & 10 & 80 & 40 & $\pi / 2$ & $5 \sim 10$ & 20 & 0.3 \\
\hline (f) & 10 & 80 & 40 & $\pi / 2$ & 7 & $15 \sim 25$ & 0.3 \\
\hline (g) & 10 & 80 & 40 & $\pi / 2$ & 7 & 20 & $0.2 \sim 0.4$ \\
\hline
\end{tabular}

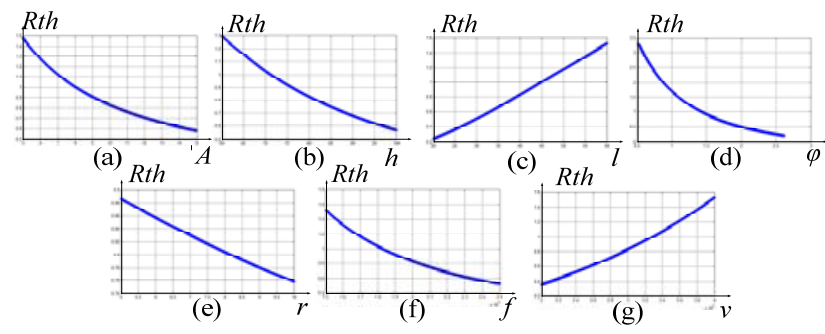

FIGURE VI. PARAMETERS AFFECTING THE THEORETICAL SURFACE ROUGHNESS.

As shown in fig. 6, an increase in dimensional parameter $l$ and nominal cutting speed $v$ will increase the theoretical surface roughness, while the other parameters have the opposite effects; dimensional parameter $l$ and tool edge radius $r$ have linear effects on the theoretical surface roughness while the other parameters are not. Another noteworthy phenomenon is shown in fig. $6(\mathrm{~g})$ that the theoretical surface roughness increases with the increase of nominal cutting speed $v$, which is opposite to the influence trend in CM. This is because lower nominal cutting speed will increase the overlapping of the elliptical locus, which can suppress the cusps and effectively reduce the surface roughness.

\section{CONCLUSIONS}

A customized tool is designed for 2D VAM. Kinematics analysis of this toolprovesthat the locus is a standard ellipse, so that the design meets the requirement of 2D VAM. Based on the kinematics and geometry analysis, a surface generation simplified model is developed for better understanding the mechanism of 2D VAM, and the theoretical surface roughness is predicted on the basis of this simplified model. The correctness of the model is verified using AdvantEdge software. All the influential parameters are listed and analysed over a wide range of values.

Although the actual surface roughness after machining is different from the theoretical one to some degree, the theoretical surface roughness model developed in this paper can provide theoretical basis for selecting suitable cutting parameters and vibration parameters in VAM. The future work is to take more practical factors, such as cutting forces fluctuation and workpiecespringback, into consideration to improve the accuracy of surface roughness model.

\section{ACKNOWLEDGEMENTS}

This work was supported in part by the National Basic Research Program of China (973 Program) granted No.2013CB035805.

\section{REFERENCES}

[1] V.I Babitsky, A.V Mitrofanov, V.V Silberschmidt. Ultrasonically assisted turning of aviation materials: simulations and experimental study, Ultrasonics, April 2004, Pages 81-86.

[2] DurulUlutan, TugrulOzel. Machining induced surface integrity in titanium and nickel alloys: A review, International Journal of Machine Tools and Manufacture, March 2011, Pages 250-280.

[3] Brehl D E, Dow T A. Review of vibration-assisted machining. Precision Engineering, 2008, 32(3): 153-172. 
[4] Zhang X, Kumar A S, Rahman M, et al. Modeling of the effect of tool edge radius on surface generation in elliptical vibration cutting. The International Journal of Advanced Manufacturing Technology, 2013: 1-8.

[5] Suraratchai, M. ; Limido, J.; Mabru, C.; Chieragatti, R. Modelling the influence of machined surface roughness on the fatigue life of aluminium alloy. International Journal of Fatigue, 2008, 30 (12): 21192126.

[6] GiDae Kim, Byoung Gook Loh. An ultrasonic elliptical vibration cutting device for micro $\mathrm{V}$-groove machining: Kinematical analysis and micro $\mathrm{V}$-groove machining characteristics, Journal of Materials Processing Technology, July 2007, Pages 181-188. 